\title{
A novel electrochemical sensor for non-invasive monitoring of lithium levels in mood disorders
}

\author{
Francesca Criscuolo*, Irene Taurino, Sandro Carrara, Giovanni De Micheli \\ Swiss Federal Institute of Technology (EPFL), Lausanne, Switzerland \\ * Email: francesca.criscuolo@epfl.ch
}

\begin{abstract}
Lithium is the main drug for the treatment of mood disorders. Due to its narrow therapeutic window, Therapeutic Drug Monitoring (TDM) is a norm during therapy in order to avoid adverse effects. Consequently, patients are obliged to frequent check-ups in hospitals to determine their serum concentration and optimize accordingly the drug dose.

Wearable sensors have attracted a growing interest in the research community in recent years owing to their promising impact in personalized healthcare. In particular, sweat diagnosis has seen an enormous expansion and is currently entering the market thanks to the large availability and simple collection of this fluid.

In this paper a novel approach for non-invasive decentralized monitoring of lithium drug concentration through sweat analysis is proposed for the first time. An all-solid-state IonSelective Electrode (ISE) with a nanostructured Solid-Contact (SC) is used to detect lithium ions in sweat. The sensor offers near-Nernstian behaviour $(57.6 \pm 2.1 \mathrm{mV} /$ decade $)$ in the concentration range of interest. In addition, it shows fast response (15-30 s), good reversibility and small potential drift over time. A wide pH stability window (pH 4-12) is also proved.
\end{abstract}

Index terms - bipolar disorder; sweat diagnosis; lithium drug; drug monitoring; personalized healthcare; wearable electrochemical sensors

\section{INTRODUCTION}

Lithium salts represent the oldest yet effective drugs to control maniac episodes and reduce the risk of suicide of psychiatric patients suffering from Bipolar Disorder (BD) [1]. BD affects every year 60 million people worldwide (WHO). In U.S. $2.6 \%$ of the adult population suffers from this serious disease (National Institute of Mental Health). BD is a potentially mortal brain disorder, which cannot be cured, but only kept under control with specific drugs. People with BD experience episodes of maniac and depressed mood, separated by periods of normal mood. Maniac events can induce unusually intense emotion, insomnia or hypersomnia, aboulia, excessive weight changes and suicidal thoughts [2], [3]. The risk of suicide is as high as $30 \%$ [4]. Therefore, after diagnosis, there is an urgent need of stabilization therapy with lithium compounds [5].

The main drawback related to lithium drug is its narrow therapeutic window (0.5-1.5 mM) [6]. Poisoning can occur if the serum concentration overcomes the maximum safe concentration $(1.5 \mathrm{mM})$. This can have severe consequences on patients' health, including ataxia, myoclonic twitching, rowsiness, and chronic toxicity that can lead to irreversible damages to liver, kidneys and brain [7]. It can eventually

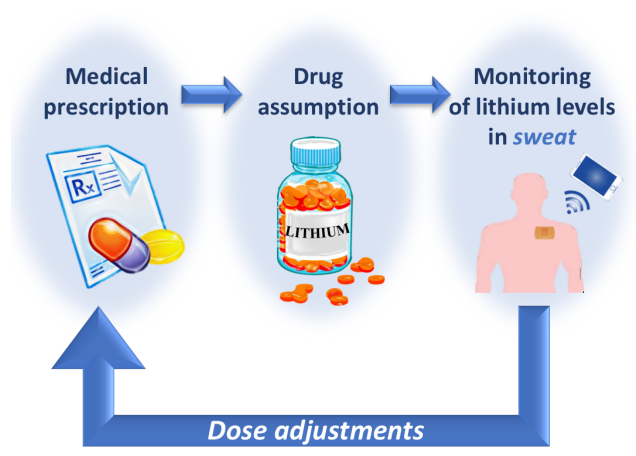

Fig. 1. The concept of Therapeutic Drug Monitoring (TDM) of lithium in sweat.

cause death. Individual variability, dietary variations and interaction with other medicines also have a strong effect on the determination of the optimum dose [7].

Consequently, Therapeutic Drug Monitoring (TDM) is essential to determine the right therapy for each patient [8]. This is typically done at the beginning of the treatment or after any change in the dose by monitoring lithium concentration in serum at least every week (standardized $12 \mathrm{~h}$ or $24 \mathrm{~h} \mathrm{Li}^{+}$serum concentration). Only in absence of any complications it is possible to decrease the check-ups frequency. On the contrary, if some abnormalities are found, the time interval between two subsequent analyses should be increased accordingly. During maintenance serum tests are in general performed every three months [1].

Lithium quantification in clinical laboratories is generally determined by complex and expensive techniques that need to be performed by qualified staff. The most common methods are atomic absorption spectrometry, flame emission photometry or conventional Ion-Selective Electrodes (ISEs) [9], but also photometry [10], [11] and colorimetry [6] techniques have been investigated. In this regards, electrochemical sensors based on all-solid-state ISEs represent a promising alternative thanks to their low cost and simplicity of measurements. These advantages make them an ideal candidate for the realization of light portable devices that would allow the remote monitoring of lithium serum concentration. It is evident that a point-of-care system for TDM would avoid 
frequent check-ups in hospitals, thus significantly improving the life quality of the patients. However, only a few examples have been reported in literature so far. A paper-based portable ISE with carbon nanotubes as SC has been demonstrated to measure lithium in serum [12]. A miniaturized $\mathrm{Li}^{+}$ISE with improved selectivity has been developed by using a graphite composite for transduction [13]. These devices certainly offer new interesting insights, however some major drawbacks still need to be overcome. These include laborious fabrication, toxicity, biocompatibility and reproducibility.

Lithium drug is absorbed in the gastrointestinal tract and then distributed to extracellular fluids and tissues [14]. It is then eliminated through kidneys, sweat and saliva. It has been demonstrated by chromatographic measurements that there is a correlation between the amount of lithium extracted in sweat and the one present in serum [15]-[17]. In particular, the concentration in sweat is about 3 times higher the one in blood. The development of a portable device to measure lithium concentration in sweat could be really helpful to remove the need of frequent medical visits for personalized therapy. Sweat offers several advantages over serum: it is readily accessible without the need of syringes and of invasive treatments, it is highly abundant and can be even reproduced artificially following specific standards. A scheme of the proposed TDM of lithium in sweat is given in Figure 1. This is based on the use of a wearable patch for direct perspiration analysis.

Wearable sensors have received a great interest in recent years as a non-invasive method for health monitoring in different biofluids, including sweat, tears and saliva [18][20]. A lot of research is currently focusing on the first one as a potential candidate to substitute blood analysis because of the several advantages discussed earlier. Both ions and other metabolites have been used as sweat biomarkers for different purposes. Electrochemistry is the most investigated sensing method because of good sensitivity, small sample volume, low cost, simple design, easy integration into microfabrication processes for large-scale production and straightforward signal interpretation. The most prominent examples are the fully integrated sweat band described in Nature [21] and the RFID sensor patch for sodium monitoring that appeared in IEEE Spectrum [22].

In this paper we demonstrate for the first time the possibility to monitor lithium concentration through perspiration analysis (Fig. 11. The sensor is based on previously developed nanostructured Solid-Contacts (SCs) [23], [24]. We are able to measure lithium concentration in sweat, with nearNernstian response $(57.6 \pm 2.1 \mathrm{mV} /$ decade $)$ in the range of interest. Good reversibility, small drift and wide $\mathrm{pH}$ stability ( $\mathrm{pH} 4-12$ ) have also been proved. These results open up new interesting perspectives towards a non-invasive decentralized management of lithium drug therapies.

\section{EXPERIMENTAL METHODS}

\section{A. Material}

$\mathrm{NaCl}$ and $\mathrm{NH}_{4} \mathrm{Cl}$ were purchased from Applichem $\mathrm{GmbH}$ (Germany). DL-Lactic acid, pyruvic acid and urea were pur- chased from Acros (United States). Uric acid was purchased from Alfa Aesar (United States). All other chemicals were purchased from Merck (Germany).

\section{B. ISM fabrication}

After the electrochemical cleaning of the electrodes [23], platinum nanostructures were electrodeposited on platinum electrodes with an active area of $12.56 \mathrm{~mm}^{2}$ (Dropsens, Spain) using an Autolab Potentiostat controlled by Nova Software (Metrohm, Switzerland), as described elsewhere [23]. $1 \mathrm{wt} \%$ Li Ionophore VI, $0.7 \mathrm{wt} \%$, Potassium tetrakis(4chlorophenyl)borate, $28.00 \mathrm{wt} \%$ Poly(vinyl chloride) high molecular weight, and $70.3 \mathrm{wt} \%$ 2-Nitrophenyl octyl ether for $100 \mathrm{mg}$ of mixture were dissolved in $1 \mathrm{ml}$ of Tethraydrofuran (THF). A volume of $10 \mu \mathrm{l}$ of membrane cocktail was drop-casted on the nanostructured electrodes. These were then kept for 24 hours at dark to allow proper drying. A conditioning step of 24 hours in a $0.003 \mathrm{M} \mathrm{LiCl}$ solution was performed prior to each measurement.

\section{Artificial Perspiration}

Artificial sweat was prepared according to the composition reported in Table I] [25].

TABLE I

COMPOSITION OF THE ARTIFICIAL PERSPIRATION SOLUTION USED IN THIS WORK.

\begin{tabular}{|c|c|}
\hline Compound & Concentration \\
\hline $\mathrm{NaCl}$ & $0.34 \mathrm{M}$ \\
\hline $\mathrm{NH}_{4} \mathrm{Cl}$ & $0.33 \mathrm{M}$ \\
\hline Acetic acid & $0.08 \mathrm{M}$ \\
\hline DL-Lactic acid & $0.20 \mathrm{M}$ \\
\hline Ascorbic acid & $10 \mu \mathrm{M}$ \\
\hline Glucose & $0.17 \mathrm{mM}$ \\
\hline Uric acid & $59 \mu \mathrm{M}$ \\
\hline Pyruvic acid & $0.18 \mathrm{mM}$ \\
\hline Glutamic acid & $0.37 \mathrm{mM}$ \\
\hline Urea & $10 \mathrm{mM}$ \\
\hline
\end{tabular}

\section{Electrochemical characterization}

All potentiometric measurements were performed using an Autolab potentiostat (Metrohm, Switzerland) controlled by a Nova 1.11 software in a two-electrodes cell setup. A double junction Sension+ 5044 Reference Electrode (Hach, United States) for use with ISEs was employed in all measurements.

\section{EXPERIMENTAL RESULTS AND DISCUSSION}

In our recent work we develop and compare different nanostructured SCs for all-solid-state ISEs with a one-step electrodeposition of noble metals [24]. This technology offers several advantages over carbon-based SCs and conductive polymers, including fast deposition, biocompatibility and non-toxicity. We proved that these systems have enhanced analytical capabilities in aqueous solutions. In this paper, for the first time we use these sensors to monitor lithium levels in sweat. The great performance of the fabricated devices in 


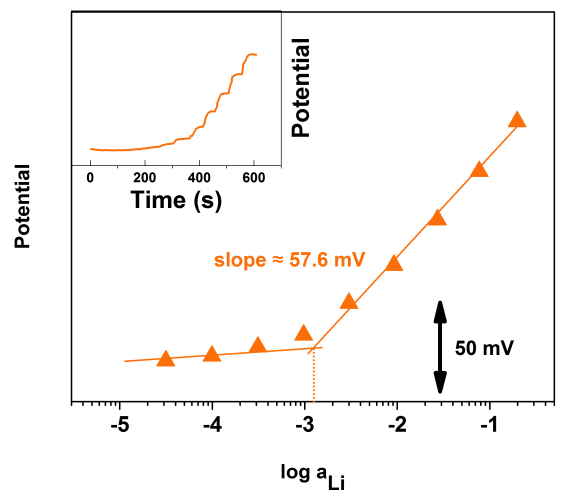

Fig. 2. Calibration curve of $\mathrm{aii}^{+} \mathrm{SC}$-ISE in sweat. The inset shows the corresponding time trace.

TABLE II

ANALYTICAL PARAMETERS IN SWEAT OF A $\mathrm{Li}^{+}$ISES BASED ON NANOSTRUCTURED SCS.

\begin{tabular}{|c|c|c|}
\hline & This work & Literature \\
\hline $\begin{array}{c}\text { sensitivity } \\
{[\mathrm{mV} / \text { decade] }}\end{array}$ & $57.6 \pm 2.1$ & $\begin{array}{c}58.7 \pm 0.8[24] \\
\text { (aqueous solution) }\end{array}$ \\
\hline $\begin{array}{c}\text { LOD } \\
{[\mathrm{M}]}\end{array}$ & $(13.5 \pm 2.1) \times 10^{-4}$ & $\begin{array}{c}5 \times 10^{-6}[13] \\
\text { (aqueous solution) }\end{array}$ \\
\hline $\begin{array}{c}\text { Response time } \\
{[\mathrm{s}]}\end{array}$ & $15-30$ & $\begin{array}{c}15-30[24] \\
\text { (aqueous solution) }\end{array}$ \\
\hline $\mathrm{pH}$ stability & $4-12$ & $\begin{array}{c}4-12[12] \\
\text { (serum) }\end{array}$ \\
\hline
\end{tabular}

this biological fluid is demonstrated, proving the feasibility of lithium drug monitoring through perspiration analysis.

An example of a typical calibration curve obtained in a perspiration solution is given in Figure 2. The corresponding time trace is given in the small inset. It is possible to observe that very stable and smooth time responses were obtained, with sharp steps after every subsequent lithium addition.

A summary of all analytical parameters and statistics is given in table $\Pi$ in comparison with the best values reported in literature. It is possible to notice that the slope of the calibration curve is $57.6 \pm 2.1 \mathrm{mV} / \mathrm{dec}$ ade, which demonstrate the near-Nernstian behaviour of the sensor. The higher standard deviation with respect to [24] can be explained by considering that in the present paper the calibration is performed in sweat, in the presence of more interactions in solution. The Limit Of Detection (LOD) is computed as the intersection of the linear portions of the calibration curve, as prescribed by IUPAC definitions [26]. Although higher with respect to the ones reported in literature for aqueous solutions, this value is still in the concentration window of interest for lithium drug in sweat, that is around 3 times the corresponding amount in serum. In addition, the response time in sweat does not increase with respect to the one obtained in simple $\mathrm{LiCl}$ aqueous solutions.

The strongest interference in $\mathrm{Li}^{+}$measurements is typically due to sodium ions because of the similar dimensions of this element. The high selectivity of the nanostructured

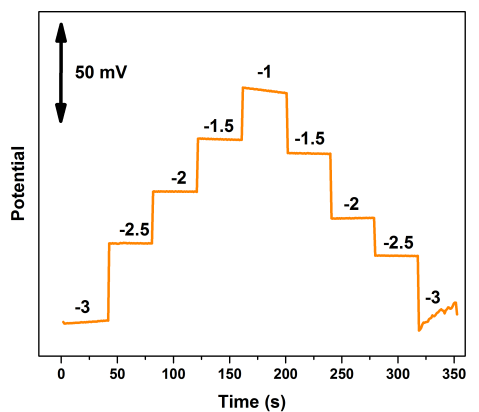

Fig. 3. Reversed calibration in the range $10^{-3}-10^{-1} \mathrm{M}$ of a $\mathrm{Li}^{+}$SC-ISE in sweat.

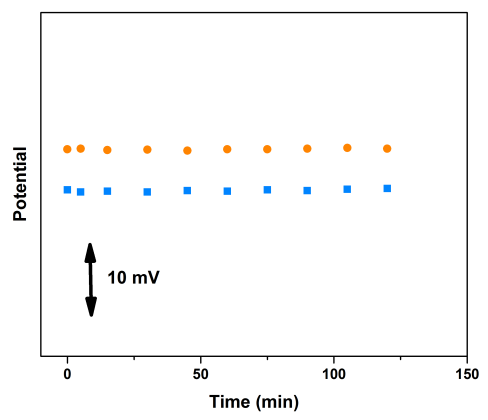

Fig. 4. Sensor stability over time when exposed to sweat with $0.01 \mathrm{M} \mathrm{LiCl}$. The two curves correspond to the responses of two distinct electrodes.

sensors used in this work was already demonstrated in aqueous solution in [24] towards all most important ions $\left(\mathrm{Na}^{+}\right.$, $\mathrm{K}^{+}, \mathrm{Ca}^{2+}, \mathrm{Mg}^{2+}, \mathrm{NH}^{4+}$ ) using both the Separate Solution Method (SSM) and the Fixed Interference Method (FIM). The quasi-Nernstian calibration obtained in the present study in the presence of a large amount of sodium ions and of several other compounds in sweat confirms our previous findings.

An important element to allow proper measurements in sweat is sensor stability over sweat $\mathrm{pH}$, since the acidity of perspiration is subjected to personal and environmental variability. The typical sweat $\mathrm{pH}$ value ranges from 4.5 to 7 [27]. The stability window versus $\mathrm{pH}$ of the sensor was measured by exposing the sensor to sweat at a constant lithium concentration of $10^{-2} \mathrm{M}$, but with different acidity. The sensor response is stable in the window of $\mathrm{pH} 4$ 12 , thus proving the suitability of the fabricated ISE for sweat analysis. This property is extremely important to allow simple data interpretation and offers an important advantage over other $\mathrm{pH}$-sensitive techniques.

The potential shown by the sensor when exposed to a constant concentration of lithium ions in sweat was compared to the value obtained by the calibration curve to analyze the predictive capability of the sensor. A percentage difference smaller than $4 \%$ was always found, thus corroborating the good stability of the sensor. 
The dynamic response of the sensor in the perspiration solution was investigated by performing a reversed calibration in the range $10^{-3}-10^{-1} \mathrm{M}$. The resulting curve is reported in Figure 3. It is possible to see that the fabricated all-solid-state ISE offer an exceptional potential stability and reversibility in sweat. Moreover, the small potential drift is evident also from the potential measurements over a prolonged period of time when the sensors is placed in perspiration solution with a constant lithium concentration (Fig. 4). A concentration of $0.01 \mathrm{M} \mathrm{LiCl}$ in sweat was used for this experiment.

Based on these results, the integration of this sensor in a wearable smart patch for sweat analysis will certainly offer a new promising and non-invasive approach for homemonitoring of lithium drug therapy directly on the skin.

\section{CONCLUSION}

In this paper, the novel idea of monitoring lithium drug concentration by non-invasive means through sweat analysis directly on the skin is proposed. The feasibility of lithium ions detection in perspiration solution is proved. A quasi-Nernstian $(57.6 \pm 2.1 \mathrm{mV} /$ decade $)$ calibration curve is obtained in the concentration range of interest for this application. Furthermore, we demonstrate that the fabricated sensors offer good reversibility and $\mathrm{pH}$ stability ( $\mathrm{pH} 4-12$ ) in sweat. A very short response time (15-30 s) and small potential drift are also achieved. The integration of these sensors in a wearable smart patch will certainly represent an important step towards a better personalized healthcare and a new non-invasive way for decentralized monitoring of lithium drug therapy for people suffering from BD.

\section{ACKNOWLEDGMENT}

This research is supported by H2020 ERC 2014 ADG 669354 CyberCare.

\section{REFERENCES}

[1] R. W. Licht, "Lithium : Still a Major Option in the Management of Bipolar Disorder Acute Antimanic Actions of Lithium," CNS Neurosci. Ther, vol. 18, pp. 219-226, 2012.

[2] K. A. Kaplan and A. G. Harvey, "Behavioral Treatment of Insomnia in Bipolar Disorder," Am. J. Psychiatry, vol. 170, no. 7, pp. 716-720, 2013.

[3] V. Umamaheswari, A. Avasthi, and G. S. Risk, "Risk factors for suicidal ideations in patients with bipolar disorder," Bipolar disord., vol. 16, pp. 642-651, 2014.

[4] M. Pompili, M. Innamorati, M. Raja, G. Ducci, G. Angeletti, D. Lester, P. Girardi, R. Tatarelli, and E. D. Pisa, "Suicide risk in depression and bipolar disorder : Do impulsiveness-aggressiveness and pharmacotherapy predict suicidal intent ?" Neuropsychiatr. Dis. Treat., vol. 4, no. 1, pp. 247-255, 2008.

[5] A. Muneer, "Staging Models in Bipolar Disorder : A Systematic Review of the Literature," Clin. Psychopharmacol. Neurosci., vol. 14, no. 2, pp. 117-130, 2016.

[6] D. Gruson, A. Lallali, V. Furlan, A. M. Taburet, A. Legrand, and M. Conti, "Evaluation of a new lithium colorimetric assay performed on the Dade Behring Dimension $\AA$ X-pand system," Clinical Chemistry and Laboratory Medicine, vol. 42, no. 9, pp. 1066-1068, 2004.

[7] H. S. Hopkins and J. Gelenberg, "Serum lithium levels and the outcome of maintenance therapy of bipolar disorder," Bipolar disord., vol. 2, pp. 174-179, 2000.

[8] G. D. Christian, "Reagents for Lithium Electrodes and Sensors for Blood Serum," Sensors, vol. 2, pp. 432-435, 2002.
[9] B. Rumbelow and M. Peake, "Performance of a novel spectrophotometric lithium assay on a routine biochemistry analyser," Annals of Clinical Biochemistry, vol. 38, no. 6, pp. 684-686, 2001.

[10] R. H. Christenson, J. J. Mandichak, S. Hong, J. M. Augustyn, and J. C. Thompson, "Clinical performance characteristics of a new photometric lithium assay : a multicenter study," Clin. Chim. Acta, vol. 327, pp. 157-164, 2003.

[11] W. Glazer, J. Sonnenberg, M. Reinstein, and R. Akers, "A novel, point-of-care test for lithium levels: description and reliability." J. Clin. Psychiatry, vol. 65, no. 5, pp. 652-5, 2004.

[12] M. Novell, T. Guinovart, P. Blondeau, F. X. Rius, and F. J. Andrade, "A paper-based potentiometric cell for decentralized monitoring of Li levels in whole blood," Lab Chip, vol. 14, no. 7, p. 1308, 2014 [Online]. Available: http://xlink.rsc.org/?DOI=c3lc51098k

[13] F. Coldur and M. Andac, "All-Solid-State Polyvinyl Chloride Membrane Lithium-Selective Electrode with Improved Selectivity and Its Application in Serum Lithium Assay," Sensor Lett., vol. 9, no. 5, pp. 1738-1744, 2011. [Online]. Available: http://openurl.ingenta.com/content/xref?genre=article $\{\&\}$ issn $=1546-198 X\{\&\}$ volume $=9\{\&\}$ issue $=5\{\&\}$ spage $=1738$

[14] A. Amdisen, "Serum level monitoring and clinical pharmacokinetics of lithium," Clinical pharmacokinetics, vol. 2, no. 2, pp. 73-92, 1977.

[15] E. Miller, R. Pain, and P. Skripal, "Sweat lithium in manic depression," Br J Psychiatry, vol. 133, 1978.

[16] j. W. Jefferson, J. H. Greist, and P. J. Clahnaz, "Effect of strenous exercise on serum lithium level in man," Am J Psychiatry, vol. 139, no. December, 1982.

[17] B. Leboulanger, J. M. Aubry, G. Bondolfi, R. H. Guy, and M. B. Delgado-Charro, "Lithium monitoring by reverse iontophoresis in vivo," Clinical Chemistry, vol. 50, no. 11, pp. 2091-2100, 2004.

[18] A. Mena-Bravo and M. D. Luque de Castro, "Sweat: A sample with limited present applications and promising future in metabolomics," Journal of Pharmaceutical and Biomedical Analysis, vol. 90, pp. 139-147, 2014. [Online]. Available: http://dx.doi.org/10.1016/j.jpba. 2013.10.048

[19] G. Matzeu, L. Florea, and D. Diamond, "Advances in wearable chemical sensor design for monitoring biological fluids," Sens. Actuators B, vol. 211, pp. 403-418, 2015. [Online]. Available: http://dx.doi.org/10.1016/j.snb.2015.01.077

[20] A. J. Bandodkar and J. Wang, "Non-invasive wearable electrochemical sensors: A review," Trends in Biotechnology, vol. 32, no. 7, pp. 363371, 2014. [Online]. Available: http://dx.doi.org/10.1016/j.tibtech. 2014.04.005

[21] W. Gao, S. Emaminejad, H. Y. Y. Nyein, S. Challa, K. Chen, A. Peck, H. M. Fahad, H. Ota, H. Shiraki, D. Kiriya, D.-H. Lien, G. A. Brooks, R. W. Davis, and A. Javey, "Fully integrated wearable sensor arrays for multiplexed in situ perspiration analysis," Nature, vol. 529, no. 7587, pp. 509-514, 2016. [Online]. Available: http://www.nature.com/doifinder/10.1038/nature16521

[22] J. Heikenfeld, "Let them see you sweat," IEEE Spectr., vol. 51, no. 11, pp. 46-63, 2014.

[23] I. Taurino, G. Sanzó, F. Mazzei, G. Favero, G. De Micheli, and S. Carrara, "Fast synthesis of platinum nanopetals and nanospheres for highly-sensitive non-enzymatic detection of glucose and selective sensing of ions," Sci. Rep., vol. 5, no. October, p. 15277, 2015. [Online]. Available: http://www.nature.com/articles/srep15277

[24] F. Criscuolo, I. Taurino, F. Stradolini, S. Carrara, and G. De Micheli, "Highly-stable $\mathrm{Li}+$ ion-selective electrode based on noble metal nanostructures as solid-contacts," Submitted for publication to Anal. Chim. Acta, 2018.

[25] T. Kilic, V. Brunner, L. Audoly, and S. Carrara, "Smart e-Patch for drugs monitoring in schizophrenia," 2016 IEEE International Conference on Electronics, Circuits and Systems, ICECS 2016, pp. 57-60, 2017.

[26] E. Lindner and Y. Umezawa, "Performance evaluation criteria for preparation and measurement of macro- and microfabricated ionselective electrodes (IUPAC Technical Report)," Pure Appl. Chem., vol. 80, no. 1, pp. 85-104, 2008.

[27] A. J. Bandodkar, V. W. S. Hung, W. Jia, G. Valdés-Ramírez, J. R. Windmiller, A. G. Martinez, J. Ramírez, G. Chan, K. Kerman, and J. Wang, "Tattoo-based potentiometric ion-selective sensors for epidermal pH monitoring," The Analyst, vol. 138, no. 1, pp. 123-128, 2013. [Online]. Available: http://xlink.rsc.org/?DOI=C2AN36422K 